\title{
Prevalência de anemia ferropriva no Brasil: uma revisão sistemática
}

\author{
Prevalence of iron-deficiency anemia in Brazil: a systematic review
}

Regina Esteves Jordão ${ }^{1}$ Júlia Laura D. Bernardi², Antônio de Azevedo Barros Filho ${ }^{3}$

\section{RESUMO}

Objetivo: Revisar os estudos de prevalência de anemia no Brasil publicados entre janeiro de 1996 e janeiro de 2007.

Fontes de dados: Por meio de revisão sistemática nas bases de dados eletrônicas Medline e Lilacs, foram selecionados títulos científicos publicados no intervalo de onze anos referentes à prevalência de anemia no Brasil em crianças menores de cinco anos de idade. Foram excluídos artigos de revisão, relatos de caso e trabalhos que relacionaram anemia a outras doenças e ao período gestacional. Para a análise comparativa das variáveis categóricas de interesse nos artigos encontrados, realizou-se o teste do qui-quadrado e o teste exato de Fisher, levando-se em conta o nível de significância estatística de $5 \%(p<0,05)$.

Síntese dos dados: Os dados medianos encontrados para a prevalência de anemia foram de $53 \%$, confirmando valores estimados pela Organização Mundial da Saúde e considerados elevados. Nos 53 artigos analisados, a variável que se associou de forma significante à anemia foi a idade da criança $(p=0,012)$.

Conclusões: Os estudos mostram alta prevalência de anemia, principalmente em crianças menores de dois anos. No entanto, os trabalhos foram realizados em creches, visitas domiciliares e Unidades Básicas de Saúde, indicando a necessidade de pesquisas com amostras de base populacional.

Palavras-chave: anemia ferropriva; lactente; prevalência; revisão; deficiência de ferro.

\section{ABSTRACT}

Objective: To conduct a systematic review on the prevalence of anemia due to iron-deficiency in Brazil from January 1996 to January 2007.

Data sources: A systematic review was conducted in electronic databases (Medline and Lilacs) in an eleven-year interval to verify the prevalence of iron deficiency anemia in children who were less than five years of age in Brazil. Reviews, case reports and studies related to anemia during pregnancy and anemia caused by others diseases were excluded. In order to describe the categorical variables according to the selected articles, the chi-square and Fisher exact tests were used. The significance level adopted was $5 \%(p<0.05)$.

Data synthesis: The median prevalence level of anemia was $53 \%$, which is considered a high prevalence rate by the World Health Organization. Among the 53 analyzed studies, the age of the children was the variable strongly associated with anemia $(p=0.012)$.

Conclusions: A high prevalence of anemia, mostly in children less than two years of age, was observed in this review. However, most studies were carried out in day care centers or in Basic Health Care Units or were obtained by home interviews, suggesting that future research should focus populational studies.

Key words: anemia, iron-deficiency; infant; prevalence; review; iron deficiency.
Instituição: Departamento de Pediatria da Faculdade de Ciências Médicas da Universidade Estadual de Campinas (FCM-Unicamp), Campinas, SP, Brasil

'Mestre pelo Curso de Pós-graduação em Saúde da Criança e do Adolescente do Departamento de Pediatria da FCM-Unicamp, Campinas, SP, Brasil

2Doutora em Saúde da Criança e do Adolescente da FCM-Unicamp, docente da Faculdade de Nutrição do Centro de Ciências da Vida da Pontifícia Universidade Católica de Campinas (PUC-Campinas), Campinas, SP, Brasil ${ }^{3}$ Professor Doutor Associado do Departamento de Pediatria da FCMUnicamp, Campinas, SP, Brasil
Endereço para correspondência:

Antônio de Azevedo Barros Filho

Rua Tessália Vieira de Camargo, 126 - Cidade Universitária

Caixa Postal 6111

CEP: 13083-887 - Campinas/SP

E-mail: abarros@fcm.unicamp.br

Recebido em: 11/6/08

Aprovado em: 18/8/08 


\section{Introdução}

A anemia é definida pela Organização Mundial da Saúde $(\mathrm{OMS})^{(1)}$ como "um estado em que a concentração de hemoglobina do sangue é anormalmente baixa em consequiência da carência de um ou mais nutrientes essenciais, qualquer que seja a origem dessa carência". Já a anemia por deficiência de ferro resulta de longo período de balanço negativo entre a quantidade de ferro biologicamente disponível e a necessidade orgânica desse oligoelemento ${ }^{(1)}$.

A anemia por deficiência de ferro é a mais comum das carências nutricionais, com maior prevalência em mulheres e crianças, principalmente nos países em desenvolvimento. Crianças entre seis e 24 meses apresentam risco duas vezes maior para desenvolver a doença do que aquelas entre 25 e 60 meses $^{(2)}$. Considerada um sério problema de Saúde Pública, a anemia pode prejudicar o desenvolvimento mental e psicomotor, causar aumento da morbimortalidade materna e infantil, além da queda no desempenho do indivíduo no trabalho e redução da resistência às infecçõos ${ }^{(3,4)}$.

$\mathrm{O}$ aumento da prevalência da anemia ferropriva em crianças pode ser decorrente das mudanças nos hábitos alimentares, que acompanham a transição nutricional no país. No Brasil, a tendência do aumento da anemia em pré-escolares foi evidenciada por dois estudos nos quais a prevalência da doença passou de 35,6\% na década de 1980, para 46,9\% na década de 1990, no município de São Paulo(5), e de 19,3\% para $36,4 \%$, na Paraíba ${ }^{(6)}$.

Devido à sua elevada prevalência e às suas conseqüências, o combate à anemia ferropriva é uma das prioridades para os profissionais responsáveis pelo planejamento de Programas de Nutrição em Saúde Públicaa ${ }^{(7)}$, encontrando respaldo político no compromisso social assumido pelo Brasil de reduzir a anemia por carência de ferro ${ }^{(8)}$. O Ministério da Saúde tornou obrigatória a fortificação das farinhas de milho e trigo com ferro e ácido fólico, por serem alimentos de fácil acesso a população e não terem alterações de suas características organolépticas no processo de fortificação, além de ser economicamente viável ao país. A partir de 1998, foi implantado o Programa Nacional de Suplementação Medicamentosa de ferro aos grupos de risco (crianças de seis a 18 meses, gestantes e mulheres no pós-parto) ${ }^{(8)}$.

No Brasil, não há levantamento nacional da prevalência de anemia, somente estudos em diferentes regiões, que mostram alta prevalência da doença, estimando-se que cerca de 4,8 milhões de pré-escolares sejam atingidos pela doença ${ }^{(9)}$. Apesar da inexistência de estudos nacionais abrangentes, dados regionais têm demonstrado elevada prevalência de anemia no Brasil, em todas as idades e níveis socioeconômicos. Portanto, diante do exposto, esta revisão teve como objetivo sistematizar os artigos publicados no período de onze anos sobre a prevalência de anemia no Brasil, na faixa etária que compreende crianças menores de cinco anos de idade.

\section{Métodos}

Nesta revisão, buscaram-se artigos indexados nas bases eletrônicas National Library of Medicine (Medline, USA) e Literatura Latino-Americana e do Caribe em Ciências da Saúde (Lilacs, Brasil), publicados em língua portuguesa e inglesa, entre janeiro de 1996 e janeiro de 2007. A revisão foi restrita aos estudos publicados com crianças menores de cinco anos de idade, por ser esta a faixa etária mais acometida pela deficiência de ferro em países em desenvolvimento.

Os descritores utilizados foram: "anemia", "ferro", "deficiência de ferro" e "Brasil" (isoladamente ou sob a forma combinada) e também na língua inglesa, de 1996 a 2007 (ano de publicação), sendo localizados 256 artigos. Estudos de revisão, experimentais com animais, artigos abrangendo população com mais de cinco anos de idade, estudos relacionando anemia com outras doenças e com o período gestacional, pesquisas em forma de tese não publicadas, artigos em duplicidade nas bases de dados e relatos de caso foram excluídos, totalizando 53 artigos para análise nesta revisão (Tabela 1).

Os artigos selecionados foram avaliados, mantendo a terminologia dos autores da pesquisa, de acordo com o ano de estudo, a região geográfica brasileira em que foram realizados, tipo de estudo, idade da criança (zero a 72 meses), tamanho amostral, percentual de prevalência de anemia de acordo com os critérios estabelecidos pela $\mathrm{OMS}^{(1,3)}$ (considerandose valores de hemoglobina abaixo de $11 \mathrm{~g} / \mathrm{dL}$ como anemia e valores inferiores a $9,5 \mathrm{~g} / \mathrm{dL}$ como anemia grave), tipo de exame sanguíneo referido no estudo (HemoCue, cianometahemoglobina, hemograma e sistema automatizado) e o local da coleta (Unidade Básica de Saúde (UBS), creche, escola, hospital ou visita domiciliar), conforme Tabela 2.

Para a discussão dos dados e interpretação das pesquisas, levou-se em consideração o ano de publicação do artigo e o período de estudo em que foi realizado o trabalho, pois essa questão pode alertar para mudanças ocorridas dentro do intervalo "ano da pesquisa - ano de publicação". Alguns estudos - 15 no total - não deixaram explícito o período de 
Tabela 1 - Resultados da pesquisa na literatura

\begin{tabular}{|c|c|c|}
\hline \multicolumn{2}{|c|}{ Base de dados pesquisada } & \multirow[t]{2}{*}{$\begin{array}{l}\text { Número de artigos } \\
\text { encontrados }\end{array}$} \\
\hline Lilacs (descritores) & & \\
\hline & Anemia & 3.445 \\
\hline & Brasil & 1.226 \\
\hline & Anemia+Brasil+ferro & 277 \\
\hline & 1996 a 2007 & 204 \\
\hline \multicolumn{3}{|l|}{ Medline (key words) } \\
\hline & Anemia & 135.140 \\
\hline & Brasil/Brazil & $16.117 / 33.281$ \\
\hline & Anemia+Brazil+iron+from 1996 to 2007 & 52 \\
\hline \multicolumn{3}{|l|}{ Critérios de exclusão } \\
\hline & Artigos de revisão e/ou revisão sistemática & 17 \\
\hline & Estudos experimentais & 11 \\
\hline & Artigos incluindo população maior de cinco anos de idade & 28 \\
\hline & Artigos incluindo população com outros tipos de anemia & 14 \\
\hline & $\begin{array}{l}\text { Artigos relacionando anemia com doenças (neoplasias, doença } \\
\text { pulmonar, entre outras) }\end{array}$ & 52 \\
\hline & Artigos relacionando anemia e gestação & 24 \\
\hline & Artigos sobre relato de caso & 4 \\
\hline & Artigos em forma de tese - não publicados & 18 \\
\hline & Artigos em duplicidade & 33 \\
\hline & Período antes de janeiro de 1996 e após janeiro de 2007 & 2 \\
\hline & Total & 203 \\
\hline \multicolumn{3}{|l|}{ Artigos incluídos } \\
\hline & Lilacs & 35 \\
\hline & Medline & 18 \\
\hline Total de artigos av & ados & 53 \\
\hline
\end{tabular}

coleta de dados, não informando a variável ano de estudo. No entanto, estes não foram excluídos, por serem importantes na análise dos demais critérios avaliados.

Além disso, foram consultados documentos de instituições governamentais abordando o tema "anemia", tais como os do Ministério da Saúde ${ }^{(9)}$, da $\mathrm{OMS}^{(1,3)}$ e dos Fundos das Nações Unidas para a infância (Unicef) ${ }^{(4,8)}$.

Os dados foram compilados no programa computacional Microsoft Office Excel e analisados por meio de comparação das variáveis categóricas de interesse com o percentual do valor mediano da prevalência, categorizados em $<50 \%$ e $\geq 50 \%$ de anemia, e pela mediana da análise comparativa das variáveis numéricas com o percentual de anemia. Para tal, empregou-se o teste do qui-quadrado e o exato de Fisher, adotando-se o nível de significância de $5 \%(p<0,05)$.

\section{Síntese dos dados}

Os artigos analisados permitiram apontar a situação da anemia no Brasil em um período de onze anos, o que con- tribui para verificar as condições dessa doença na população infantil. Houve maior número de pesquisas (no total de 24) realizadas sobre o tema entre os anos de 1995 e 1999 , mostrando que o interesse pelo assunto e a necessidade de respostas aumentaram e que estudos transversais e de intervenção foram necessários (Tabela 2).

A década de 1990 repercutiu positivamente sobre os estudos e as ações de intervenção direcionadas para anemia no Brasil e no mundo, após a resolução da OMS, em reunião realizada na cidade de Roma, na Itália, buscando, para o século 21 , soluções para diminuir a prevalência dessa doença ${ }^{(10)}$.

A região geográfica brasileira com maior número de pesquisas (28 no total) foi a Sudeste, com 46,4\% de estudos apresentando prevalência de anemia $\geq 50 \%$ da amostra. Por outro lado, $70 \%$ dos estudos (dez no total) descreveram a presença de anemia em mais da metade das crianças avaliadas nas regiões Norte e Centro-Oeste, mostrando que, apesar destas serem regiões menos investigativas em relação à presença da doença, houve alta prevalência da mesma. 


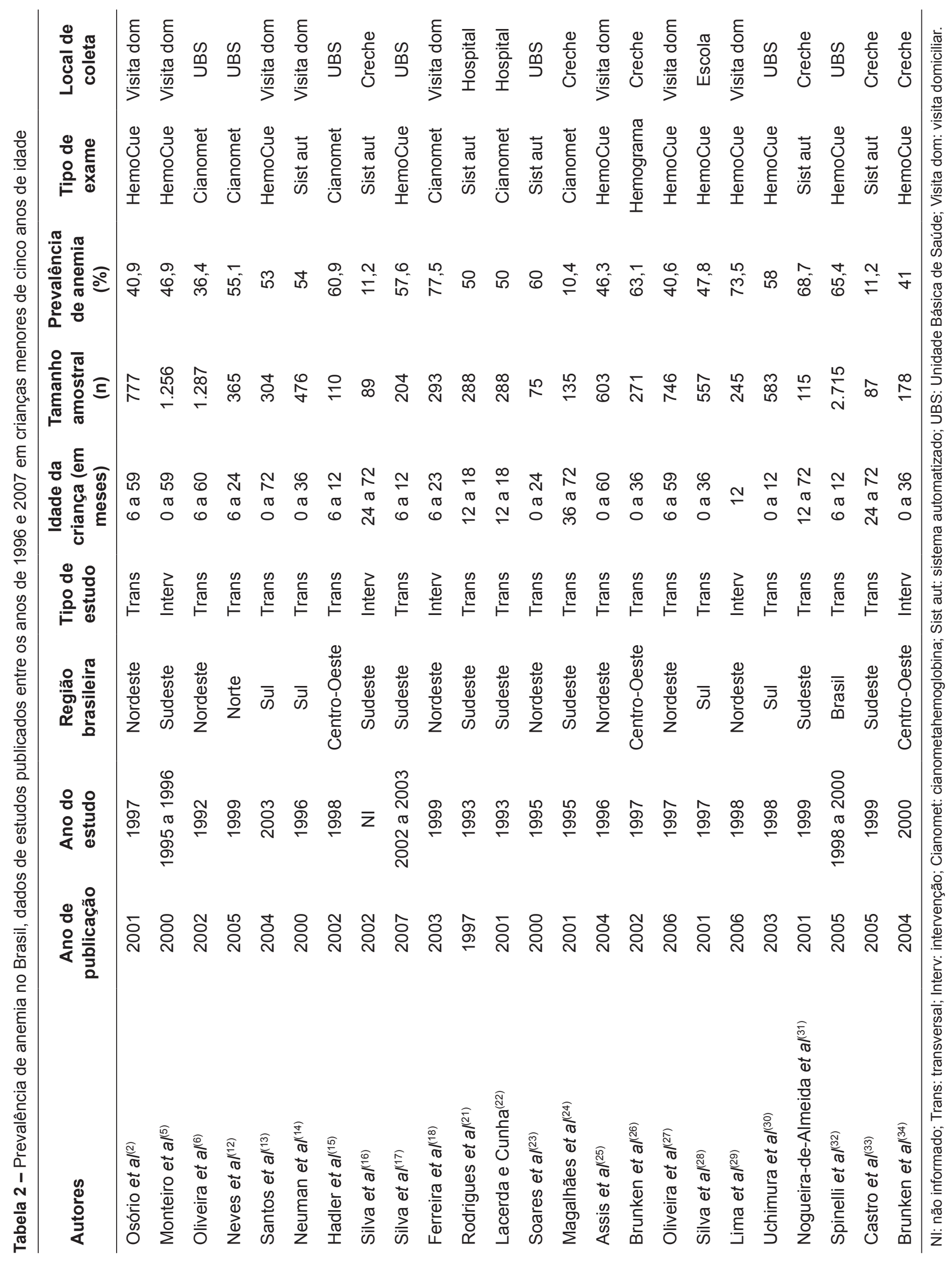




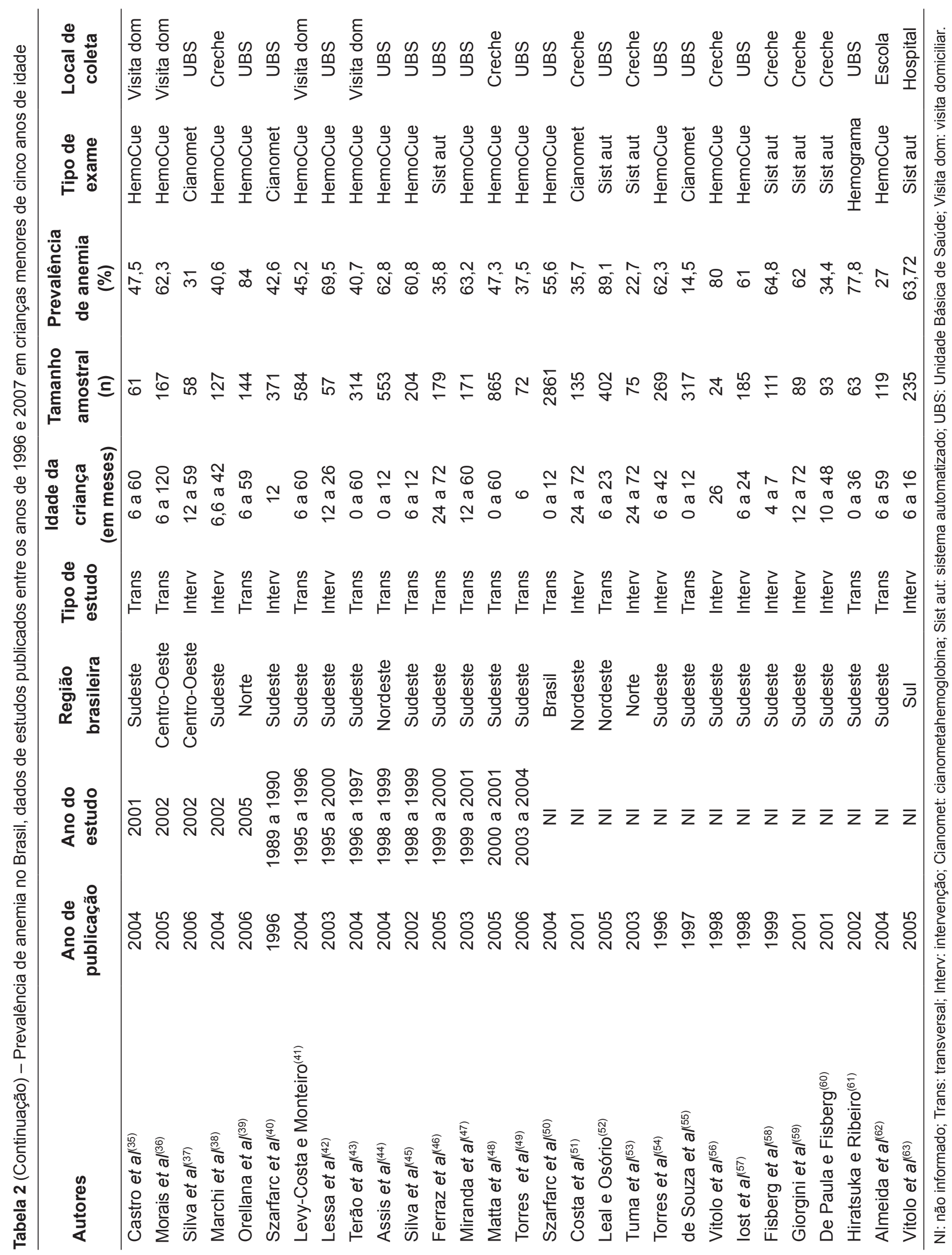


$\mathrm{Na}$ região Nordeste, um estudo ${ }^{(2)}$ com 777 crianças de seis a 59 meses avaliou as áreas metropolitana, urbana (interior) e rural e encontrou $40,9 \%$ de anemia. A zona rural apresentou maior prevalência $(51,4 \%)$ de anemia do que as regiões urbana $(35,9 \%)$ e metropolitana $(39,6 \%)$ $(\mathrm{p}<0,001)$. O estudo demonstrou que os fatores associados à maior prevalência de anemia na região rural foram: baixo consumo de ferro heme devido às condições de pobreza, rede de distribuição pobre em alimentos de origem animal, condições ambientais desfavoráveis para o plantio de frutas e verduras, saneamento básico precário e alto risco de parasitoses ${ }^{(11)}$.

$\mathrm{Na}$ Região Norte, um estudo transversal ${ }^{(12)}$ realizado em Belém, Pará, avaliou 365 crianças de seis a 24 meses de idade atendidas em um serviço de saúde pública e encontrou prevalência de 55,1\% de anemia, sendo que a deficiência de ferro associou-se à não utilização de fórmula láctea fortificada introduzida como primeiro leite ou em substituição ao leite materno durante o processo de desmame, na faixa etária entre seis e 12 meses de idade e na faixa de renda inferior a um salário mínimo.

Dados da Região Sul mostraram que tanto as crianças moradoras da periferia quanto as da área urbana estão sujeitas à anemia. Dois estudos realizados no Sul do país - um no município de Pelotas ${ }^{(13)}$ e outro em Criciúma ${ }^{(14)}$ - encontraram prevalência da doença de 53 e $54 \%$, respectivamente, sendo que o primeiro avaliou 304 crianças com idade menor que seis anos moradoras da região periférica da cidade e o segundo investigou 476 menores que três anos na região urbana.

Em relação ao tipo de estudo, o método mais empregado foi o transversal (66\%). A UBS foi o local em que ocorreu maior número de coleta de dados, totalizando 22 estudos. Tal fato pode ter influenciado os resultados devido às características da população que recorre ao serviço público. O número de pesquisas realizadas em creches e em visita domiciliar foi semelhante (14 e 12, respectivamente).

$\mathrm{Na}$ presente revisão, a faixa etária foi analisada em cada estudo por meio de frequência da anemia, a cada 12 meses de idade da criança, conforme descrito na Tabela 3. A idade das crianças avaliadas variou de zero a cinco anos, sendo que, nas publicações realizadas com lactentes menores de um ano, a análise comparativa da prevalência de anemia mostrou-se estatisticamente significante, com mediana de $55,3 \%(\mathrm{dp}=15,1 ; p=0,012)$. As crianças menores de cinco anos são mais propensas a ter anemia e os estudos avaliados confirmaram esse fato. Nas pesquisas realizadas com crianças menores de um ano de idade, estas foram identificadas como grupo de risco para a doença ${ }^{(15-17)}$.

A prevalência de $77,5 \%$ de anemia na região Nordeste, no município de Caruaru, Ceará, foi descrita por Ferreira et $a^{(18)}$. O estudo avaliou crianças de seis a 23 meses de idade, com o intuito de verificar a efetividade da aplicação do sulfato ferroso em doses semanais no Programa Saúde da Família no agreste pernambucano. O estudo evidenciou que o grupo etário em questão foi o mais exposto ao risco de anemia e houve redução da doença para 40,3\%, mostrando que a estratégia realizada com doses de $5 \mathrm{mg}$ de ferro elementar por semana durante cerca de 24 semanas é eficaz no controle das formas graves de anemia e na redução de sua prevalência.

Os estudos analisados nesta revisão incluíram tamanho amostral que variou entre $<100$ a $>500$ crianças até cinco anos, sendo que $81,2 \%$ dos estudos na faixa de amostragem entre 200 e 499 apresentaram prevalência de anemia $\geq 50 \%$.

A maioria das pesquisas $(52,8 \%)$ realizou o procedimento de coleta sanguínea por punção capilar, utilizando hemoglobinômetro portátil digital do tipo HemoCue ${ }^{\circledR}$. Apenas 3,8\% dos estudos verificaram a prevalência de anemia por hemograma. O uso do HemoCue possui especificidade e sensibilidade suficientes para detectar níveis alterados de hemoglobina, facilitando o diagnóstico imediato da população avaliada e permitindo o encaminhamento mais eficaz e rápido para tratamento. Assim, embora o HemoCue possa resultar em diagnósticos falsos-negativos ${ }^{(19)}$, é um método validado para pesquisas de campo ${ }^{(20)}$.

\section{Considerações finais}

Os 53 artigos analisados nesta revisão compreenderam a somatória de 20.952 crianças avaliadas. Os dados medianos encontrados para a prevalência de anemia foram de $53 \%$. No território nacional, as regiões de maior prevalência da doença foram a Norte e a Centro-Oeste. Um número elevado de pesquisas concentrou-se no Sudeste. No entanto, para obter um conhecimento mais acurado da situação, há necessidade de estudos que utilizem amostras representativas com base populacional e adequação do cálculo amostral. 
Tabela 3 - Análise comparativa das variáveis categóricas com a porcentagem de anemia categorizada em <50\% e $\geq 50 \%$

\begin{tabular}{|c|c|c|c|}
\hline \multirow[t]{2}{*}{ Variáveis categóricas } & \multicolumn{2}{|c|}{$\begin{array}{l}\text { Número de artigos de acordo com a prevalência } \\
\text { de anemia }\end{array}$} & \multirow[t]{2}{*}{ Valor de $p^{*}$} \\
\hline & $<50 \%$ & $\geq \mathbf{5 0} \%$ & \\
\hline \multicolumn{4}{|l|}{ Ano de estudo } \\
\hline 1989 a 1994 & 2 & 2 & 0,705 \\
\hline 1995 a 1999 & 10 & 14 & \\
\hline 2000 a 2005 & 6 & 4 & \\
\hline \multicolumn{4}{|l|}{ Regiões brasileiras } \\
\hline Norte, Centro-Oeste & 3 & 7 & 0,436 \\
\hline Nordeste & 5 & 5 & \\
\hline Sudeste & 15 & 13 & \\
\hline Sul & 1 & 4 & \\
\hline \multicolumn{4}{|l|}{ Tipo de estudo } \\
\hline Intervenção & 9 & 9 & 0,621 \\
\hline Transversal & 15 & 20 & \\
\hline \multicolumn{4}{|l|}{ Idade da criança } \\
\hline Contempla 12 meses & 17 & 27 & 0,012 \\
\hline Contempla 24 meses & 20 & 14 & \\
\hline Contempla 36 meses & 21 & 10 & \\
\hline Contempla 48 meses & 18 & 6 & \\
\hline Contempla 60 meses & 12 & 5 & \\
\hline \multicolumn{4}{|l|}{ Tamanho da amostra } \\
\hline$<100$ & 7 & 5 & 0,055 \\
\hline 100 a 199 & 6 & 7 & \\
\hline 200 a 499 & 3 & 13 & \\
\hline$\geq 500$ & 8 & 4 & \\
\hline \multicolumn{4}{|l|}{ Local da coleta } \\
\hline Creche & 9 & 5 & 0,122 \\
\hline Unidades Básicas de Saúde & 6 & 16 & \\
\hline Visita domiciliar & 7 & 5 & \\
\hline Escola/hospital & 2 & 3 & \\
\hline \multicolumn{4}{|l|}{ Tipo de exame } \\
\hline Cianometahemoglobina & 6 & 4 & 0,543 \\
\hline HemoCue & 13 & 15 & \\
\hline Hemograma & 0 & 2 & \\
\hline Sistema automatizado & 5 & 8 & \\
\hline
\end{tabular}

*nível de significância: $p<0,05$.

\section{Referências bibliográficas}

1. Organização Mundial de Saúde. Lucha contra la anemia nutricional, especialmente contra la carência de hierro: Informe ADI/OIEA/OMS. Série de Informes Técnicos, 580. Genebra: OMS, 1975.

2. Osório MM, Lira PI, Batista-Filho M, Ashworth A. Prevalence of anemia in children 6-59 months old in the state of Pernambuco, Brazil. Rev Panam Salud Publica 2001;10:101-7.

3. World Health Organization. Iron deficiency anemia: assessment, prevention and control. A guide for programme managers. WHO/NDH/01.3. Geneve: WHO/NHD, 2001.

4. Unicef. The state of the world's children. Oxford University Press: New York, 1998.
5. Monteiro CA, Szarfarc SC, Mondini L. Secular trends in childhood in the city of São Paulo, Brazil (1984-1996). Rev Saude Publica 2000;34 (6 Suppl): S62-72.

6. Oliveira RS, Diniz Ad Ada S, Benigna MJ, Miranda-Silva SM, Lola MM, Gonçalves MG et al. Magnitude, geographic distribution and trends of anemia in preschoolers, Brazil. Rev Saude Publica 2002;36:26-32.

7. Batista Filho M, Barbosa NP. Pró-memória: alimentação e nutrição no Brasil: 1974-1984. Brasília: INAN; 1985.

8. Unicef/Fundação Dalmo Giacometti/Embrapa. Encontro de trabalho sobre estratégias e planos de ação para a fortificação de alimentos no Brasil (vitamina A, ferro e iodo): relatório final. Brasília: Unicef, 1997. 
9. Brasil - Ministério da Saúde. Coordenação Geral da Política de Alimentação e Nutrição. Oficina de trabalho "Carências Nutricionais: Desafio para Saúde Pública". Brasília: Ministério da Saúde, 2004.

10. World Health Organization, FAO. International Conference on Nutrition. Rome, Final report. Geneva: FAO/WHO, 1992.

11. Secretaria de Saúde do Estado de Pernambuco. II Pesquisa Estadual de Saúde e Nutrição. Saúde, nutrição, alimentação e condições sócio-econômicas no Estado de Pernambuco. Recife: Governo do Estado de Pernambuco/Instituto Materno-Infantil de Pernambuco/Departamento de Nutrição, Universidade Federal de Pernambuco, 1998.

12. Neves MB, da Silva EM, de Morais MB. Prevalence and factors associated with iron deficiency in infants treated at a primary care center in Belém, Pará, Brazil. Cad Saude Publica 2005;21:1911-8.

13. Santos I, César JA, Minten G, Valle N, Neumann NA, Cercato E. Prevalence of anemia and associated factors among children under six years of age in Pelotas, South Brazil. Rev Bras Epidemiol 2004;7:403-15.

14. Neumann NA, Tanaka OY, Szarfarc SC, Guimarães PR, Victora CG. Prevalence and risk factors for anemia in Southern Brazil. Rev Saude Publica 2000;34:56-63.

15. Hadler MC, Juliano Y, Sigulem DM. Anemia in infancy: etiology and prevalence. J Pediatr (Rio J) 2002;78:321-6.

16. Silva MR, Castro TG, Costa NB, Ferreira CL, Franceschini SC, Leal PF et al. Efeito de uma bebida láctea fermentada e fortificada com ferro sobre o estado nutricional de ferro em pré-escolares.Viçosa-MG. Nutrire 2002;23:23-32.

17. Silva DG, Priore SE, Franceschini S do C. Risk factors for anemia in infants assisted by public health services: the importance of feeding practices and iron supplementation. J Pediatr (Rio J) 2007;83:149-56.

18. Ferreira ML, Ferreira LO, Silva AA, Batista Filho M. Effectiveness of weekly iron sulfate in the Family Health Program in Caruaru, Pernambuco State, Brazil. Cad Saude Publica 2003;19:375-81.

19. Neufeld L, García-GuerraA, Sánchez-Francia D, Newton-Sánchez O, RamírezVillalobos MD, Rivera-Dommarco J. Hemoglobin measured by Hemocue and a reference method in venous and capillary blood: a validation study. Salud Publica Mex 2002;44:219-27.

20. Brasil - Ministério da Saúde. Vigilância alimentar e nutricional (Sisvan). Orientações básicas para a coleta, o processamento, a análise de dados e a informação em serviços de saúde. Brasília: Ministério da Saúde, 2004.

21. Rodrigues CR, Motta SS, Cordeiro AA, Lacerda EM, Reichenheim ME. Prevalence of iron deficiency anemia and risk indicators in children from 12 to 18 months attended at the outpatient clinic of Instituto de Puericultura e Pediatria Martagão Gesteira. J Pediatr (Rio J) 1997;73:189-94.

22. Lacerda E, Cunha AJ. Iron deficiency anemia and nutrition in the second year of life in Rio de Janeiro, Brazil. Rev Panam Salud Publica 2001;9:294-301.

23. Soares NT, Guimarães AR, Sampaio HA, Almeida PC, Coelho RR. Nutritional status of infants in slum areas of Fortaleza, Brazil. Rev Nutr 2000;13:99-106.

24. Magalhães P, Ramalho RA, Colli C. Iron and vitamin A deficiencies: nutritional evaluation nutritional in pre-school children from the city of Viçosa (MG -Brazil). Nutrire 2001;21:41-56.

25. Assis AM, Barreto ML, Gomes GS, Prado M da S, Santos NS, Santos LM et al. Childhood anemia prevalence and associated factors in Salvador, Bahia, Brazil. Cad Saude Publica 2004;20:1633-41.

26. Brunken GS, Guimarães LV, Fisberg M. Anemia in children under 3 years of age in public day care centers. J Pediatr (Rio J) 2002;78:50-6.

27. Oliveira MA, Osório MM, Raposo MC. Hemoglobin level and anemia in children in the State of Pernambuco, Brazil: association with socioeconomic and food consumption factors. Cad Saude Publica 2006;22:2169-78.

28. Silva LS, Giuglian ER, Aerts DR. Prevalence and risk factors for anemia among children in Brazil. Rev Saude Publica 2001;35:66-73.

29. Lima AC, Lima MC, Guerra MQ, Romani SA, Eickmann SH, Lira PI. Impact of weekly treatment with ferrous sulfate on hemoglobin level, morbidity and nutritional status of anemic infants. J Pediatr (Rio J) 2006;82:452-7.

30. Uchimura TT, Szarfarc SC, Latorre MR, Uchimura NS, Souza SB. Anemia e peso ao nascer. Rev Saude Publica 2003;37:397-403.

31. Nogueira-de-Almeida CA, Ricco RG, Del Ciampo LA, de Souza AM, Dutrade-Oliveira JE. Growth and hematological studies on Brazilian children of low socioeconomic level. Arch Latinoam Nutr 2001;51:230-5.
32. Spinelli MG, Marchioni DM, Souza JM, Souza SB, Szarfarc SC. Risk factors for anemia among 6- to 12-month-old children in Brazil. Rev Panam Salud Publica 2005;17:84-91.

33. Castro TG, Novaes JF, Silva MR, Costa NM, Franceschini SC, Tinoco AL et al Characteristics of dietary intake, socioeconomic environment and nutritional status of preschoolers at public kindergartens. Rev Nutr 2005;18:321-30.

34. Brunken GS, Muniz PT, Silva SM. Weekly iron supplementation reduces anemia prevalence by 1/3 in preschool children. Rev Bras Epidemiol 2004;7:210-9.

35. Castro TG, Campos FM, Priore SE, Coelho FM, Campos MT, Franceschini SC et al. Health and nutrition of children, 0 to 60 -month old, in an agrarian-reform settlement, Vale do Rio Doce, MG, Brazil. Rev Nutr 2004;17:167-76.

36. Morais MB, Alves GM, Fagundes-Neto U. Nutritional status of Terena indian children from Mato Grosso do Sul, Brazil: follow up of weight and height and current prevalence of anemia. J Pediatr (Rio J) 2005;81:383-9.

37. Silva AP, Vitolo MR, Zara LF, Castro CF. Effects of zinc supplementation on 1- to 5-year old children. J Pediatr (Rio J) 2006;82:227-31

38. Marchi RP, Szarfarc SC, Rodrigues JE. Consumption of fortified rice in profilaxis of iron deficiency. Nutrire 2004;28:53-64.

39. Orellana JD, Coimbra CE Jr, Lourenço AE, Santos RV. Nutritional status and anemia in Surui Indian children, Brazilian Amazon. J Pediatr (Rio J) 2006;82:383-8.

40. Szarfarc SC, Berg G, Santos AL, Souza SB, Monteiro CA. Prevention of anemia in the first year of life in health centers of Santo André, São Paulo. J Pediatr (Rio J) 1996;72:329-34.

41. Levy-Costa RB, Monteiro CA. Cow's milk consumption and childhood anemia in the city of São Paulo, southern Brazil. Rev Saude Publica 2004;38:797-803.

42. Lessa Ado C, Devincenzi MU, Sigulem DM. Comparison of nutritional status of low-income children in the second year of life before and after primary health care intervention. Cad Saude Publica 2003;19:505-14.

43. Terão SM, Puccini RF, Silva EM, Pedroso GC, Silva NN. Prevalência de anemia em crianças residentes no município do Embu (São Paulo), 1996-7. Rev Paul Pediatr 2004;22:7-14.

44. Assis AM, Gaudenzi EN, Gomes G, Ribeiro R de C, Szarfarc SS, Souza SB Hemoglobin concentration, breastfeeding and complementary feeding in the first year of life. Rev Saude Publica 2004;38:543-51.

45. Silva DG, Franceschini S do C, Priore SE, Ribeiro SM, Szarfarc SC, Souza SB et al. Iron deficiency anemia in 6 to 12-month-old infants attended at the public health service of Viçosa, Minas Gerais, Brazil. Rev Nutr 2002;15:301-8.

46. Ferraz IS, Daneluzzi JC, Vannucchi H, Jordão AA Jr, Ricco RG, Del Ciampo LA et al. Prevalence of iron deficiency and its association with vitamin A deficiency in preschool children. J Pediatr (Rio J) 2005;81:169-74.

47. Miranda AS, Franceschini SC, Priori SE, Euclydes MP, Araújo RM Ribeiro SM et al. Iron deficiency anemia and nutritional status of children aged 12 to 60 months in the city of Viçosa, MG, Brazil. Rev Nutr 2003; 16:163-9.

48. Matta IE, Veiga GV, Baião MR, Santos MM, Luiz RR. Anemia in children under five years old attended at public day care centers from Rio de Janeiro, Brazil. Rev Bras Saude Mater Infant 2005;5:349-57.

49. Torres MA, Braga JA, Taddei JA, Nóbrega FJ. Anemia in low-income exclusively breastfed infants. J Pediatr (Rio J) 2006;82:284-7.

50. Szarfarc SC, de Souza SB, Furumoto RA, Brunken GS, Assis AM, Gaudenz EM et al. Hemoglobin concentration in children from birth to one year of age. Cad Saude Publica 2004;20:266-74.

51. Costa MJ, Terto AL, Santos LM, Rivera MA, Moura LS. Supplementation with West Indian cherry and its effects on the blood levels of vitamin $C$ and hemoglobin in preschool children. Rev Nutr 2001;14:13-20.

52. Leal LP, Osório MM. Validity and reproducibility of the clinical signs for the diagnosis of anemia in children. Cad Saude Publica 2005;21:565-72.

53. Tuma RB, Yuyama LK, Aguiar JP, Marques HO. Impact of cassava flour fortified with iron amino acid chelate on the hemoglobin level in pre-schools. Rev Nutr 2003;16:29-39.

54. Torres MA, Lobo NF, Sato K, Queiroz S de S. Fortification of fluid milk for the prevention and treatment of iron deficiency anemia in children under 4 years of age. Rev Saude Publica 1996;30:350-7.

55. de Souza SB, Szarfarc SC, de Souza JM. Anemia in the first year of life and its relation to breast-feeding. Rev Saude Publica 1997;31:15-20. 
56. Vitolo MR, Aguirre AN, Kondo MR, Giuliano Y, Ferreira N, Lopez FA. Effect of the use of iron-enriched cereal on the serum hemoglobin levels and anthropometric values of preschool children. Rev Nutr 1998;11:163-71.

57. lost C, Name JJ, Jeppsen RB, Ashmead HD. Repleting hemoglobin in iron deficiency anemia in young children through liquid milk fortification with bioavailable iron amino acid chelate. J Am Coll Nutr 1998;17: 187-94.

58. Fisberg M, Ferreira AM, Silva JV, Kliamca PE, Cardoso R, Giorgini E et al. Nova fórmula lactea infantil: papel na prevenção da anemia carencial. Pediatria (São Paulo) 1999;21:48-59.

59. Giorgini E, Fisberg M, de Paula RA, Ferreira AM, Valle J, Braga JA. The use of sweet rolls fortified with iron bis-glycinate chelate in the prevention of iron deficiency anemia in preschool children. Arch Latinoam Nutr 2001;51 (Suppl 1):S48-53.

60. de Paula RA, Fisberg M. The use of sugar fortified with iron tris-glycinate chelate in the prevention of iron deficiency anemia in preschool children. Arch Latinoam Nutr 2001;51 (Suppl 1):S54-9.

61. Hiratstuka MS, Ribeiro ZM. Diagnosis of anemia in children aged 0-3 - A need for recommendations. RSDF 2002;13:7-14.

62. Almeida AP, Zandonade E, Abrantes MM, Lamounier JA. Deficiência de ferro e anemia em crianças de Vitória, ES. Pediatria 2004;26:140-50.

63. Vitolo MR, Bortolini GA, Feldens CA, Drachler Mde L. Impacts of the 10 steps to healthy feeding in infants: a randomized field trial. Cad Saude Publica 2005;21:1448-57. 